\title{
MicroRNA-663a is downregulated in non-small cell lung cancer and inhibits proliferation and invasion by targeting JunD
}

Yi Zhang ${ }^{1}$, Xiaoman Xư ${ }^{2}$, Meng Zhang ${ }^{1}$, Xin Wang ${ }^{1}$, Xue Bai ${ }^{1}$, Hui Li ${ }^{1}$, Liang Kan ${ }^{1}$, Yong Zhou' ${ }^{1}$, Huiyan Niu ${ }^{1}$ and Ping $\mathrm{He}^{1 *}$

\begin{abstract}
Background: MicroRNA-663a expression is downregulated in several tumors. However, its functions and mechanisms in human non-small cell lung (NSCLC) cancer remain obscure. The present study aimed to identify the expression pattern, biological roles and potential mechanisms by which miR-663a dysregulation is associated with NSCLC.

Methods: We examined expression level of miR-663a in 62 cases of NSCLC tissues and 5 NSCLC cell lines by reverse transcription PCR. In vitro, gain-of-function and loss-of-function experiments were performed to examine the impact of miR-663a on proliferation, cell cycle progression and invasion of NSCLC cells. Using fluorescence reporter assays, we also explored the potential targets and possible mechanisms of miR-663a in NSCLC cells.

Results: Downregulation of miR-663a was observed in 42 of 62 of lung cancer tissues compared with paired normal tissues (mean cancer/normal value $=0.745$ ) and its downregulation correlated with nodal metastasis. Transfection of miR-663a mimic suppressed cell proliferation, cell cycle progression and invasion, with downregulation of cyclin D1, cyclin E and MMP9 in both H460 and H1299 cell lines. Transfection of miR-663a inhibitor in both H460 and $\mathrm{H} 1299$ cell lines exhibited the opposite effects. In addition, we confirmed that miR-663a could inhibit AP-1 activity and AP-1 component JunD was a direct target of miR-663a in lung cancer cells. Transfection of miR-663a mimic downregulated JunD expression. In addition, JunD siRNA treatment abrogated miR-663a inhibitor-induced expression of cyclin D1, cyclin E and MMP9. Above all, both miRNA mimic and inhibitor in two different NSCLC cell lines demonstrated that miR-663a inhibits proliferation and invasion by targeting AP-1 transcription factor JunD.
\end{abstract}

Conclusions: This study indicates that miR-663a downregulation might be associated with NSCLC progression. MiR-663a suppresses proliferation and invasion by targeting AP-1 component JunD in NSCLC cells.

Keywords: Lung cancer, miR-663a, JunD, Proliferation, Invasion

\section{Background}

Lung cancer is the leading cause of cancer-related death worldwide, and the incidence of lung cancer is increasing [1]. Overall, the 5-year survival rate has remained at $15 \%$ for the past two decades. Although targeted therapies have been established, genetic mutations causing activation of these gene products are identified

\footnotetext{
*Correspondence: hepingcmu@126.com

'Department of Geriatrics, Shengjing Hospital of China Medical University, 36 Sanhao Road, Shenyang 110004, China

Full list of author information is available at the end of the article
}

only in a limited number of cancers $[2,3]$. On the other hand, a variety of complex genetic, epigenetic, and microenvironmental factors play important roles in survival and invasion of tumor cells [4-7]. Hence, identification of these biological factors and elucidation of their regulatory pathways in governing tumor development, invasion, and metastasis is an important step toward the rational design of drugs for treatment of advanced NSCLC.

MicroRNAs (miRNAs) are a class of small non-coding RNAs, approximately $20-25$ nucleotides, which regulate gene expression post-transcriptionally. Nearly $50 \%$ of 
human miRNAs are located at fragile sites and genomic regions involved in cancers [8-10]. Emerging evidence shows that miRNA dysregulation is associated with various cancers including lung cancer $[10,11]$. Previous studies have shown that miR-663a, a member of primate-specific miRNA family, is associated with a variety of important biologic processes such as viral infection, inflammatory responses and autoimmune diseases [12, 13]. However, its role in tumor progression is controversial. miR-663a serves as a potential tumor suppressor in gastric cancer, colorectal carcinomaand acute lymphoblastic leukemia [14-17], while it acts as an oncogene in nasopharyngeal carcinoma and breast cancer $[18,19]$.

In the present study, we evaluated miR-663a expression and clinical relevance in human non-small cell lung cancer tissues. Its involvement in biological behavior and the underlying molecular mechanisms were also investigated. Our data identified miR-663a as a potential tumor suppressor in human lung nonsmall cell lung cancer.

\section{Methods \\ Samples}

Fresh samples from lung cancer and corresponding normal adjacent tissue were obtained from patients at Shengjing of China Medical University between January 2011 and November 2013 with informed consent. None of the patients in the study received any chemotherapy or radiation therapy before surgery. This study was conducted with the approval of the Ethics Committee at Shengjing Hospital of China Medical University. Written informed consent was obtained from all patients. Research carried out is in compliance with the Helsinki Declaration. Tumor samples were stored at $-80^{\circ} \mathrm{C}$ for RNA extraction (the percentage of tumor tissue was $>90 \%$ ).

\section{Cell culture, reagents and transfection}

HBE135, H1299, H157, H1395, H460 and H3255 cell lines were obtained from American Type Culture Collection (Manassas, VA, USA). Cells were cultured in RPMI-1640 (Invitrogen, Carlsbad, CA, USA) containing $10 \%$ fetal calf serum (Invitrogen, Carlsbad, CA, USA), $100 \mathrm{IU} / \mathrm{ml}$ penicillin (Sigma, St. Louis, MO, USA), and $100 \mu \mathrm{g} / \mathrm{ml}$ streptomycin (Sigma). Cells were grown on sterilized culture dishes and were passaged every 2 days with $0.25 \%$ trypsin (Invitrogen, Carlsbad, CA, USA).

A mimic negative control, miR-663a mimic, an inhibitor negative control and a miR-663a inhibitor were purchased from RiboBio (Guangzhou, China). miRNA mimic is small, double-stranded RNA molecule, designed to mimic endogenous mature miRNA molecules when transfected into cells. miRNA inhibitor is small, single-stranded RNA with chemical modification that regulate gene expression by binding to and inhibiting a specific mature miRNA. MiR-663a mimic and inhibitor were transfected using Dharmafect1 Transfection Reagent (Dharmacon Lafayette, CO, USA). Briefly, complexes containing the mimic or inhibitor were prepared according to the manufacturer's protocol and then cells were transfected with mimic negative control $(100 \mathrm{nM})$, miR-663a mimic $(100 \mathrm{nM})$, inhibitor control $(200 \mathrm{nM})$ or miR-663a inhibitor (200nM), respectively.

siGENOME SMARTpool siRNA for JunD and Nontargeting siRNA \#1 were purchased from Dharmacon (100nM per well). The cells were transfected with siRNA using the DharmaFECT $1(0.20 \mu \mathrm{L} /$ well; Dharmacon Lafayette, CO, USA) according to the manufacturer's protocol.

\section{Quantitative real-time PCR}

Total RNA was extracted from fresh tissues and cells using Trizol (Invitrogen, Carlsbad, CA, USA) according to the manufacturer's instructions. Quantitative realtime PCR was performed using SYBR Green PCR master mix (Applied Biosystems, Foster City, CA, USA) on 7900HT Fast Real-Time PCR System (Applied Biosystems, Foster City, CA, USA). Primers for miR-663a (Bulge-LoopTM miRNA qRT-PCR Primer Set for hasmiR-663a, RiboBio, Guangzhou, China) and U6 (U6 snRNA qRT-PCR Primer Set, RiboBio, Guangzhou, China) were used for PCR analysis in accordance with the manufacturer's protocol. Other primers are as followers: cyclin D1 forward, 5'-GCTGGAGGTCTGCG AGGA-3', cyclin D1 reverse, 5'-ACAGGAAGCGGTC CAGGTAGT-3, cyclin E forward, 5'-AGCCAGCCT TGGGACAATAAT-3', cyclin E reverse, 5'-GAGCCT CTGGATGGTGCAAT-3', p21 forward, 5'-CCTCATCC CGTGTTCTCCTTT-3'. p21 reverse, 5'-GTACCACCC AGCGGACAAGT-3'. CDK4 forward, 5'-CCGAAGT TCTTCTGCAGTCC-3'. CDK4 reverse, 5'-GTCGGCT TCAGAGTTTCCAC-3'. CDK6 forward, 5' -GTGACCA GCAGCGGACAAAT-3'. CDK6 reverse, 5'-CCACA GCGTGACGACCACT-3'. MMP2 forward, 5'-TGTG TTCTTTGCAGGGAATGAAT-3'. MMP2 reverse, $5^{\prime}$-T GTCTTCTTGTTTTTGCTCCAGTTA-3'. MMP9 forward, 5'-CCTCTGGAGGTTCGACGTGA-3', MMP9 reverse, 5 '-TAGGCTTTCTCTCGGTACTGGAA-3', $\beta$ actin forward, $5^{\prime}$-ATAGCACAGCCTGGATAGCAAC GTAC-3', $\beta$-actin reverse, 5'-CACCTTCTACAATG AGCTGCGTGTG-3'. A dissociation step was performed to generate a melting curve to confirm the specificity of the amplification. Each PCR analysis was performed in triplicate. The relative levels of gene expression were represented as $\Delta \mathrm{Ct}=\mathrm{Ct}$ gene $-\mathrm{Ct}$ reference, and the fold change of gene expression was calculated by the $2^{-\Delta \Delta C t}$ method. 


\section{Western blot analysis}

Total proteins from cells were extracted in lysis buffer (Pierce, Rockford, IL, USA) and quantified using the Bradford method. $50 \mu \mathrm{g}$ protein was separated by SDSPAGE. Samples were transferred to polyvinylidene fluoride membranes (Millipore, Billerica, MA, USA) and incubated overnight at $4{ }^{\circ} \mathrm{C}$ with antibody against cyclin D1(1:1000, Cell Signaling Technology, Boston, MA, USA), cyclin E (1:1000, Cell Signaling Technology, Boston, MA, USA), p21(1:1000, Cell Signaling Technology, Boston, MA, USA), CDK4(1:1000, Cell Signaling Technology, Boston, MA, USA), CDK6 (1:1000, Cell Signaling Technology, Boston, MA, USA), MMP2(1:1000, Cell Signaling Technology, Boston, MA, USA), MMP9(1:1000, Cell Signaling Technology, Boston, MA, USA) and JunD (1:1000, Cell Signaling Technology, Boston, MA, USA) and GAPDH (1:1000; Santa Cruz, CA, USA). After incubation with peroxidase-coupled anti-mouse/rabbit IgG (1:1000, Cell Signaling Technology, Boston, MA, USA) at $37{ }^{\circ} \mathrm{C}$ for $2 \mathrm{~h}$, bound proteins were visualized using ECL (Pierce, Rockford, IL, USA) and detected using a DNR BioImaging System (DNR, Jerusalem, Israel). Relative protein levels were quantified using ImageJ software.

\section{Colony formation assay}

For evaluation of colony formation, cells were transfected for $48 \mathrm{~h}$ before being seeded into 6 - $\mathrm{cm}$ cell culture dishes (800 per dish) and incubated for 14 days. The plates were washed with PBS and the colonies were stained with the Giemsa dye. The number of colonies with more than 50 cells was manually counted under the microscope.

\section{Cell cycle analysis by flow cytometry}

Cells $(500,000)$ were seeded into $6 \mathrm{~cm}$ tissue culture dishes and cultured overnight. Forty eight hours after transfection, cells were harvested, fixed in cold $70 \%$ ethanol washed with phosphate-buffered saline (PBS) and stained with $5 \mathrm{mg} / \mathrm{ml}$ propidium iodide in PBS supplemented with RNase A (Roche, Indianapolis, IN) for $30 \mathrm{~min}$ at room temperature. The cells were analyzed by flow cytometer (Becton-Dickinson and Co., San Jose, CA, USA). One-parameter histogram was plotted according to the distribution of nuclear DNA content in each cell detected by flow cytometer. Cells in each individual phase of the cell cycle were determined based on their DNA ploidy profile.

\section{Matrigel invasion assay}

Cell invasion assay was performed using 24-well transwell chambers with pore size of $8 \mu \mathrm{m}$, and the inserts were coated with $20 \mu \mathrm{l}$ Matrigel (1:3 dilution, BD Bioscience, San Jose, CA, USA). Sixty hours after transfection, cells were trypsinized, transferred to the upper matrigel chamber in $100 \mu \mathrm{l}$ serum-free medium containing $3 \times 105$ cells, and incubated for $18 \mathrm{~h}$. Medium supplemented with $10 \%$ FBS was added to the lower chamber as the chemoattractant. Then, the non-invading cells on the upper membrane surface were removed with a cotton tip, and the cells that passed through the filter were fixed in $4 \%$ paraformaldehyde and stained with hematoxylin. The experiments were performed in triplicate.

\section{Luciferase reporter assay}

Reporter gene transfection and luciferase activity assay were performed as follows: cells in confluent growth on a 24 well plate were co-transfected with the firefly luciferase reporter $(0.2 \mu \mathrm{g})$ along with the Renilla luciferase reporter $(0.02 \mu \mathrm{g})$, which was used for normalization, using Attractene reagent (Qiagen, Hilden, Germany) according to the protocols provided by the manufacturers. The reporter plasmids of AP-1 were purchased from Biotime Biotechnology, China. The pAP1-Luc contains SV40 early enhancer/promoter. The AP-1 response element was listed as follows: GGCCTAACTG GCCGGTACCG CTAGCTG ACT AATGACTAAT GACTAATGAC; CCGGATTGAC CGGCCATGGC GATCGACTGA TTACTGATTA CT GATTACTG. The luciferase activity was measured in cellular extracts using a dual luciferase reported gene assay kit (Promega, San Luis Obispo, CA, USA). The relative activity of the reporter gene was calculated by dividing the signals from firefly luciferase reporter by the signals obtained from Renilla luciferase reporter.

\section{Validation for interaction of miRNAs and Target Genes using Luciferase Reporter Assays}

A pmiR-RB-REPORT T ${ }^{\mathrm{tm}}$ vector (Ribobio Co., Guangzhou, China) was used for 3' UTR-luciferase reporter assays to detect interactions of miR-663a with JunD (Gene ID: 3727). The TargetScan Human database $6.2 \mathrm{http}: / / \operatorname{tar}-$ getscan.org/was used to identify miRNA binding sites. The Wild-type miR-663a target site in JunD 3'-untranslated region was CCCCGCC. The mutant miR-663a target site was CGGGCGC. The miR-663a mimic, miR663a inhibitor and their corresponding negative controls (Ribobio Co. Guangzhou, China) were transfected with pmiR-RB-REPORT ${ }^{\text {tw }}$-target gene UTR. Reporter assays were conducted in triplicate. The cells were cotransfected with $100 \mathrm{nM}$ mimic and $100 \mathrm{ng} / \mathrm{mL}$ reporter plasmid by attractene agent (Qiagen, Hilden, Germany) according to the manufacturer's instructions

\section{Statistical analysis}

SPSS version 11.5 for Windows was used for all statistical analyses. Student's $t$-test was used to compare densitometry data on focus numbers between control and treated cells. All p values are based on a two-sided statistical analysis and $p<0.05$ was considered to indicate statistical significance. 


\section{Results}

miR-663a expression was downregulated in NSCLC and associated with lymph lode metastasis

We examined levels of miR-663a by RT-qPCR in 62 fresh NSCLC tissue samples with paired normal lung tissues. As shown in Fig. 1a, the expression levels of miR-663a were downregulated in 42 of 62 NSCLC tissues. As shown in the boxplot (Fig. 1b), the mean level of miR-663a was lower in NSCLC tissues than in normal lung tissues (mean cancer/normal value $=0.745$ ). To determine the effect of miR-663a on tumor progression, NSCLC patients were divided into two groups, low and high expression, according to the median relative expression level of miR-663a in cancerous tissues (median cancer/normal value $=0.773$ ).

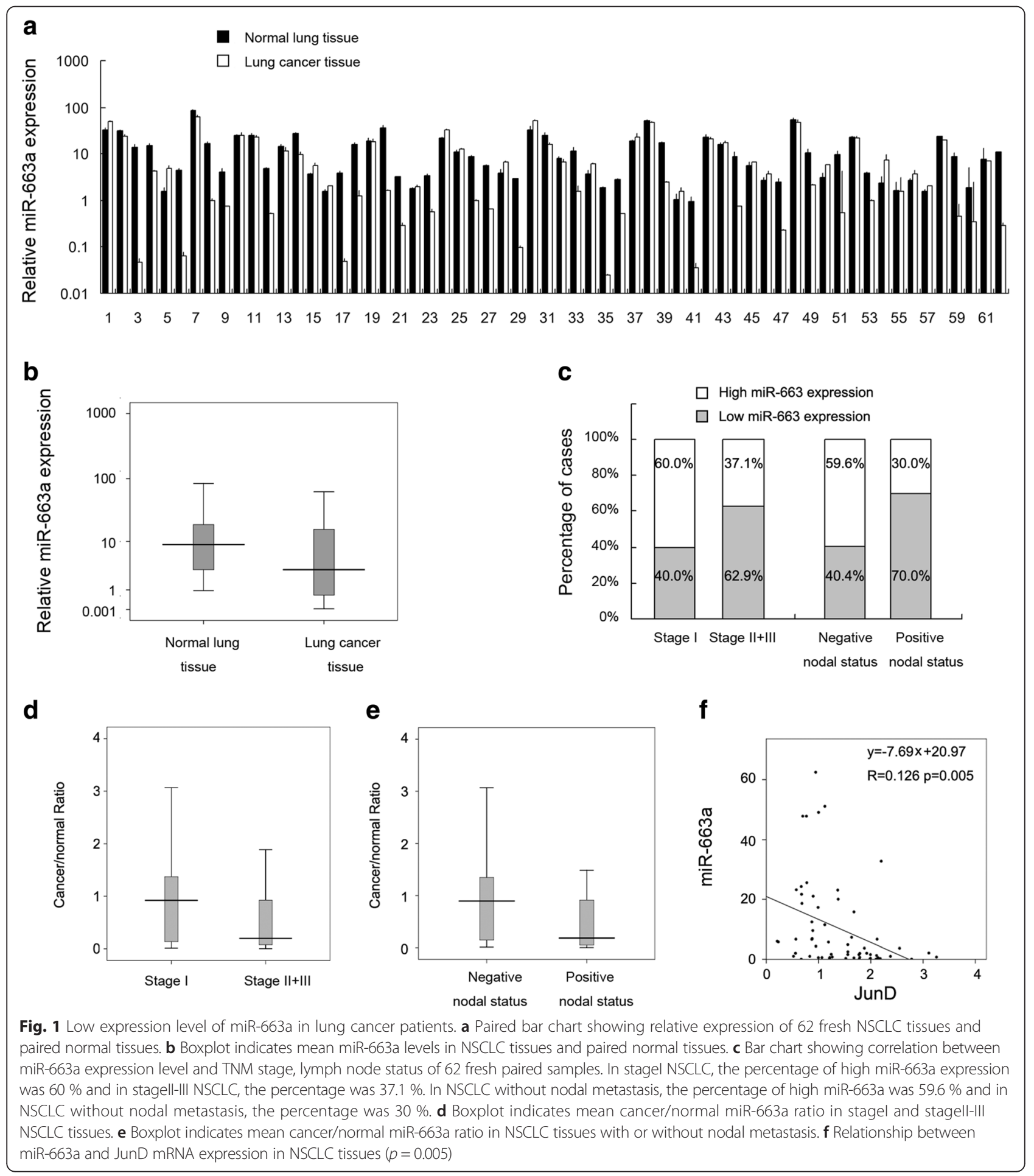


Correlations between miR-663a expression and clinicopathologic variables were shown in Table 1. Significant correlation was observed between miR-663a expression and nodal status $(p=0.0297)$ (Chi-Square test). In 20 cases presenting with positive nodal status, $14(70.0 \%)$ cases had low expression of miR-663a, while the low expression rate was $17 / 42(40.4 \%)$ in tumor samples without nodal metastasis (Fig. 1c-e). No correlation was observed between miR-663a expression and gender $(p=0.7668)$, age $(p=0.7926)$, histology type $(p=0.7978)$, stage $(p=0.0730)$ or histological grade $(p=0.3508)$.

miR-663a regulates cell proliferation and invasion in vitro We examined the expression of miR-663a in normal bronchial cell line HBE135 and 5 NSCLC cell lines: H1299 (adenocarcinoma), H3255 (adenocarcinoma), H460 (large cell carcinoma), H1395 (adenocarcinoma) and H157 (squamous cell carcinoma) using RT-qPCR. As shown in Fig. 2a, compared to the expression in HBE135, downregulation of miR-663a was observed in H157, H1395 and H460 cell lines. H3255 cell line has the same level of miR-663a as HBE135 and H1299 has

Table 1 Distribution of miR-663a status in NSCLC according to clinicopathological characteristics

\begin{tabular}{|c|c|c|c|c|}
\hline Characteristics & $\begin{array}{l}\text { Number of } \\
\text { patients }\end{array}$ & $\begin{array}{l}\text { Low miR-663a } \\
\text { expression }\end{array}$ & $\begin{array}{l}\text { High miR-663a } \\
\text { expression }\end{array}$ & $P$ \\
\hline \multicolumn{5}{|l|}{ Age } \\
\hline$<60$ & 23 & 12 & 11 & \multirow[t]{2}{*}{0.7926} \\
\hline$\geq 60$ & 39 & 19 & 20 & \\
\hline \multicolumn{5}{|l|}{ Gender } \\
\hline Male & 47 & 24 & 23 & \multirow[t]{2}{*}{0.7668} \\
\hline Female & 15 & 7 & 8 & \\
\hline \multicolumn{5}{|l|}{ Histology } \\
\hline Adenocarcinoma & 27 & 13 & 14 & \multirow[t]{2}{*}{0.7978} \\
\hline $\begin{array}{l}\text { Squamous cell } \\
\text { carcinoma }\end{array}$ & 35 & 18 & 17 & \\
\hline \multicolumn{5}{|l|}{ Differentiation } \\
\hline Well & 10 & 7 & 3 & \multirow[t]{3}{*}{0.3508} \\
\hline Moderate & 42 & 20 & 22 & \\
\hline Poor & 10 & 4 & 6 & \\
\hline \multicolumn{5}{|l|}{ TNM stage } \\
\hline I & 35 & 14 & 21 & \multirow[t]{2}{*}{0.0730} \\
\hline$\|+1\|$ & 27 & 17 & 10 & \\
\hline \multicolumn{5}{|l|}{ Tumor status } \\
\hline $\mathrm{T} 1$ & 16 & 7 & 9 & \multirow[t]{2}{*}{0.5616} \\
\hline $\mathrm{T} 2-\mathrm{T} 4$ & 46 & 24 & 22 & \\
\hline \multicolumn{5}{|l|}{ Nodal status } \\
\hline NO & 42 & 17 & 25 & \multirow[t]{2}{*}{0.0297} \\
\hline N1 - N3 & 20 & 14 & 6 & \\
\hline
\end{tabular}

relative high expression. We explored the potential effects of miR-663a on proliferation, cell cycle progression and invasion in lung cancer cells. According to the expression of miR-663a in NSCLC cell lines, we selected H460 cells for miR-663a mimic transfection and H1299 cells for miR-663a inhibitor transfection. Then we examined the efficiency of miR-663a mimic and inhibitor by real-time PCR at $48 \mathrm{~h}$ after transfection. As shown in Fig. 2a, miR-663a mimic significantly upregulated miR-663a in H460 cells and inhibitor downregulated miR-663a expression in H1299 cells. Colony formation assay showed that colony number of $\mathrm{H} 460$ cells transfected with miR-663 mimic was significantly decreased (control $596 \pm 28$ vs mimic $401 \pm 18, p<0.05$ ), while colony formation ability of H1299 transfected with miR-663a inhibitor was increased (control $171 \pm 16$ vs inhibitor $295 \pm 18, p<0.05$ ) (Fig. 2b). Cell cycle analysis showed that treatment of miR-663a mimic inhibited G1-S transtion, while miR-663a inhibitor treatment facilitated cell cycle progression (Fig. 2c).

Matrigel invasion assays were also performed, and exogenously increase of miR-663a expression significantly reduced the number of invasive cells by $41.8 \%$ in $\mathrm{H} 460$ cells (control $86 \pm 6$ vs mimic $50 \pm 3, p<0.05$ ) (Fig. 2d). The number of invasive cells significantly increased by 48.8 \% in H1299 cellstransfected with miR-663a inhibitor compared with the control cells (control $84 \pm 5$ vs inhibitor $125 \pm 9, p<0.05$ ) (Fig. 2d).

In addition, to demonstrate the underlying mechanisms of miR-663a- mediated inhibition of proliferation and invasion, weexamined expression of cell cycle and invasion related proteins, including cyclin D1, cyclin E, p21, CDK4, CDK6, MMP2 and MMP9 by realtime PCR and western blot. The results showed that miR-663a mimic transfection could downregulate cyclin D1, cyclin E and MMP9 expression, while treatment with miR-663a inhibitor upregulated these proteins (Fig. 3a and b). The effect of miR-663a on other proteins was not obvious.

Since the difference in miR-663a levels between H1299 and H460 cells is not large, we also transfected miR-663a inhibitor in H460 cells and miR-663a mimic in H1299 cells to further confirm the results. Consistent with the above findings, we found that colony number of H1299 cells transfected with a mimic was significantly decreased (control $278 \pm 20$ vs mimic $169 \pm 18, p<0.05$ ), while colony formation ability of H460 transfected with miR-663a inhibitor was increased (control $415 \pm 32$ vs mimic $581 \pm 19, p<0.05)$. Similarly, cell cycle analysis showed that treatment of miR-663a mimic in H1299 cells inhibited G1-S transtion, while miR-663a inhibitor treatment in $\mathrm{H} 460$ cells facilitated cell cycle progression. In addition, exogenously upregulation of miR-663a in H1299 cells reduced the number of invasive cells significantly (control $72 \pm 3$ vs mimic $46 \pm 4, p<0.05$ ). The 


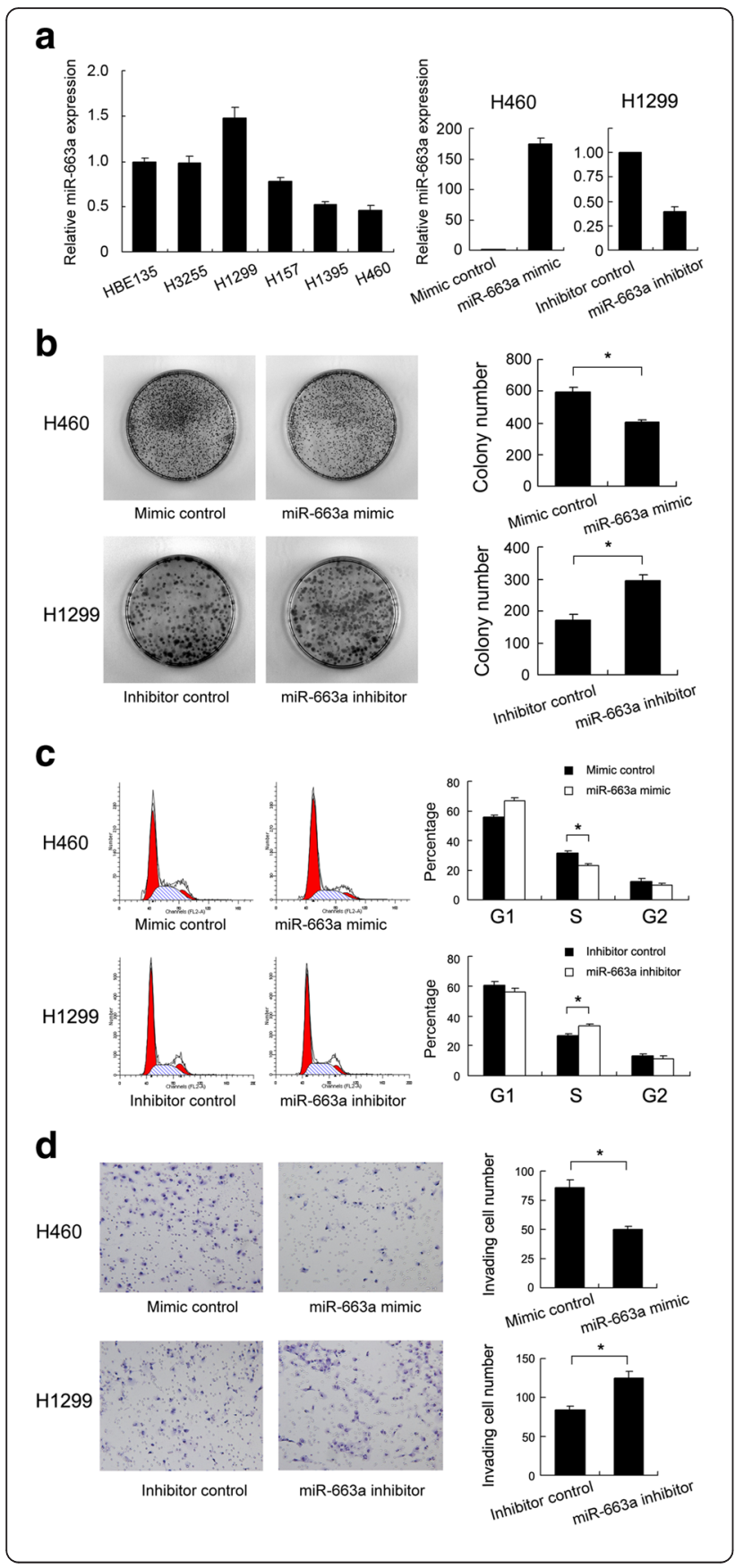

Fig. 2 Effects of miR-663a on proliferation and invasion in NSCLC cells. a Expression of miR-663a in 5 NSCLC cell lines (H1299, H3255, H460, H1395, H157) and normal bronchial cell line HBE135. miR-663a mimic significantly upregulated miR-663a level in $\mathrm{H} 460$ cells and its inhibitor downregulated miR-663a expression in H1299 cells. b Colony number of $\mathrm{H} 460$ cells transfected with miR-663a mimic was lower than cells transfected with control (control $596 \pm 28$ vs mimic $401 \pm 18, p<0.001$ ). Colony number of H1299 transfected with miR-663a inhibitor was higher than cells transfected with control (control $171 \pm 16$ vs inhibitor $295 \pm 18, p<0.001$ ). c miR-663a mimic increased G1 phase percentage and decreased $S$ phase percentage in H460 cells. miR-663a inhibitor decreased G1 phase percentage and increased S phase percentage in H1299 cells. d Matrigel invasion assay showed that invading number of $\mathrm{H} 460$ transfected with miR-663a mimic was lower than cells transfected with control (control $86 \pm 6$ vs mimic $50 \pm 3, p<0.001$ ). Invading number of H1299 cells transfected with miR-663a inhibitor was higher than cells transfected with control (control $84 \pm 5$ vs inhibitor $125 \pm 9, p<0.001$ ). ${ }^{*} p<0.05$, Error bars indicate standard deviation

number of invasive cells in $\mathrm{H} 460$ cells increased significantly when transfected with miR-663a inhibitor (control $45 \pm 3$ vs mimic $61 \pm 4, p<0.05$ ). Finally, we assessed the roles of miR-663a in the regulation of cell-cycle and cellinvasion related molecules. As expected, miR-663a inhibitor increased both protein and mRNA expression of cyclin D1, cyclin E, MMP9 and JunD in H460 cells, meanwhile miR-663a mimic showed opposite effects in H1299 cells (Additional file 1: Figure S1).

\section{miR-663a inhibits AP-1 transcription activity by targeting JunD}

Since miR-663a could downregulate cyclin D1, cyclin E and MMP9 expression, we examined several signaling pathways which could regulate these genes simultaneously. Using luciferase reporter plasmid, we found that miR-663a mimic transfection inhibited AP-1 transcription activity and miR-663a inhibitor increased AP-1 activity (Fig. 4a).

To determine potential target genes of miR-663a in NSCLC, the TargetScan Human database 6.2 was used. Among the genes in the target list, we focused on JunD which is an AP-1 component and a key mediator of tumor cell proliferation, metastasis and chemoresistance in a variety of tumors (Additional file 2: Figure S2). To evaluate whether JunD is regulated by miR-663a in vitro, we transfected H460 cells with miR-663a mimic and detected the mRNA and protein levels of JunD $48 \mathrm{~h}$ after transfection.. As shown in Fig. 4, ectopic expression of miR-663a significantly decreased both mRNA and protein expression of JunD compared to controls., indicating miR-663a as a potent regulator of JunD.

To further determine if JunD was a direct target of miR-663a, fluorescent reporter assays were performed. According to a previous study [15], the 3 '-UTR of JunD with wild-type (CCCCGCC) and mutant (CGGGCGC) 


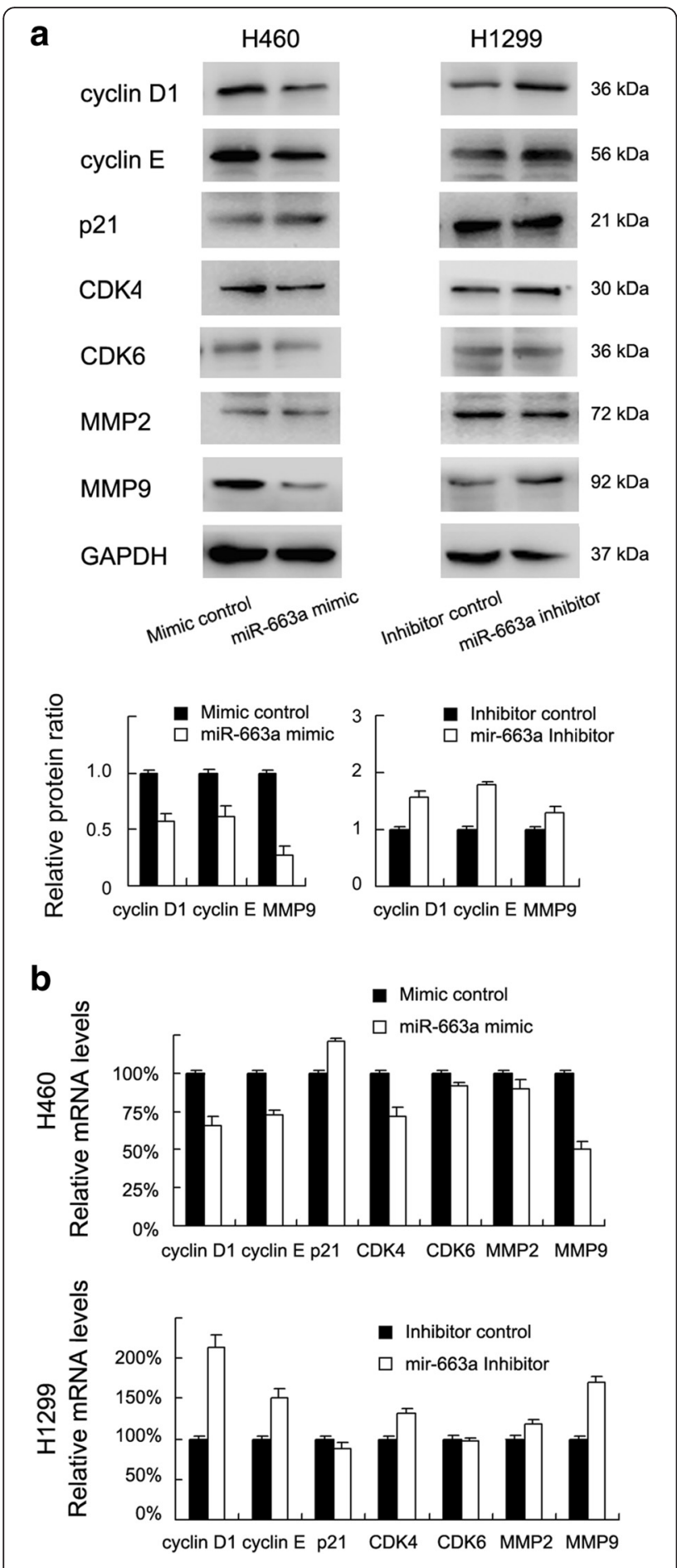

Fig. 3 miR-663a regulates mRNA and protein expression of cyclin D1, cyclin E and MMP9. a Western blot showed that cyclin D1, cyclin E and MMP9 protein levels of H460 cells treated with miR-663a mimic were lower than those of control. cyclin D1, cyclin E, MMP9 protein levels of H1299 cells treated with miR-663a inhibitor were higher than those of control. Protein quantification of protein was shown for cyclin D1, cyclin E and MMP9 in these two cell lines. b Realtime PCR analysis showed that cyclin D1, cyclin E, MMP9 mRNA expression of $\mathrm{H} 460$ cells treated with miR-663a mimic were lower than those of cells transfected with control. cyclin D1, cyclin E, MMP9 mRNA expression of $\mathrm{H} 1299$ cells treated with miR-663a inhibitor were higher than those of cells transfected with control. Experiments were repeated in triplicate. Error bars indicate standard deviation

binding sites for miR-663a was cloned into pmiR-RBREPORT vector. The ratio of fluorescence intensity for wild-type and mutant binding sites was calculated. As shown in Fig. 4c, miR-663a mimic reduced the fluorescence intensity in $\mathrm{H} 460$ cells transfected with a vector containing wild-type JunD 3'-UTR compared with controls, while no significant change was observed in cells transfected with vector containing mutant binding site. These results indicated that miR-663a binds to JunD 3'UTR region directly and downregulates JunD mRNA expression. To validate this, we analyzed the relationship between miR-663a and JunD mRNA expression in NSCLC tissues using linear regression. We found that miR-663a negatively correlated with JunD mRNA expression (Fig. 1f, $p=0.005$ ).

To confirm the role of JunD-AP1 axis in miR-663a induced inhibition of cyclin proteins and MMP9. We knocked down JunD using siRNA in H1299 cell line. Western blot and realtime PCR analysis showed that JunD depletion downregulated mRNA and protein expression of cyclin D1, cyclin E and MMP9. In addition, in JunD siRNA treated H1299 cells, miR-663a inhibitor failed to upregulate cyclin D1, cyclin E and MMP9. These results indicated the pivotal role of JunD in miR663a mediated inhibition of cell growth and invasion.

\section{Discussion}

Accumulated evidence in the literature implicates an important role of miR-663a in cancer development [14-17]. It was reported that expression of miR-663a was significantly downregulated in pediatric $A M L$ cells, which may caused by hypermethylation of the miR-663a promoter [17]. Downregulation of miR-663a was also observed in gastric cancers and introduction of miR-663a into the human gastric cancer cells suppressed proliferation and mitotic catastrophe [14]. In addition, the tumor suppressive role of miR-663a was demonstrated in colorectal cancer cells [16], prostate cancer and especially in glioblastoma, in which miR663a targeted PIK3CD and served as a prognostic biomarker [20]. However, the involvement of miR- 
a

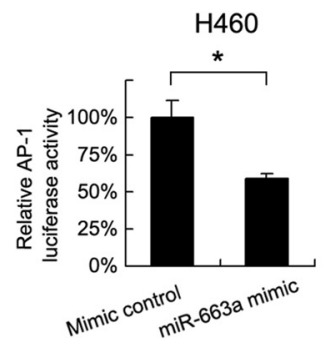

b

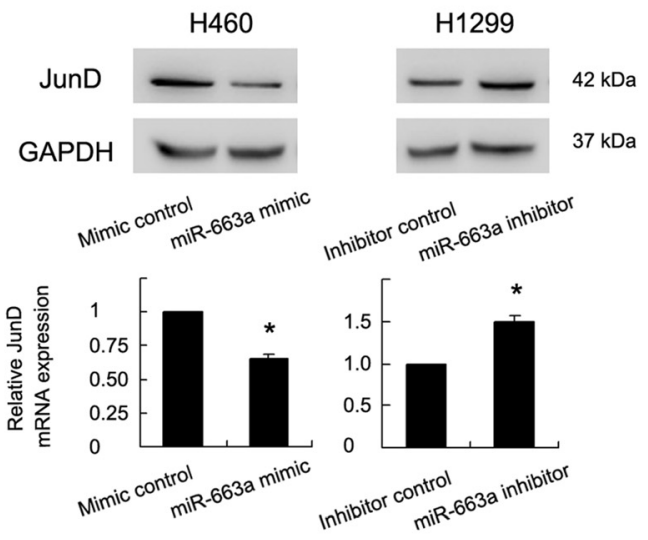

C

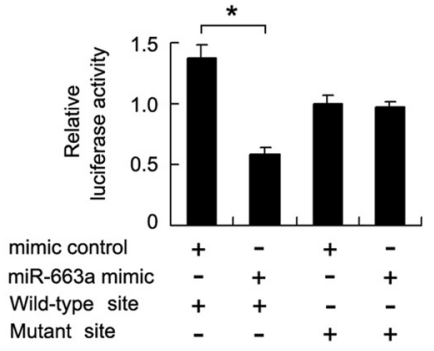

\section{d}

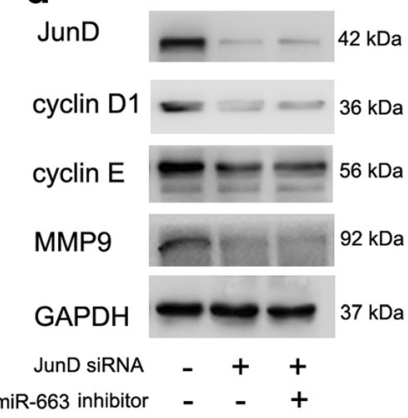

miR-663 inhibitor - _ +

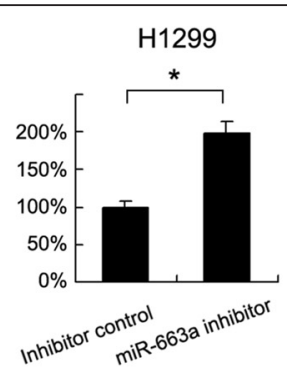

H1299

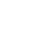


criticalinvasion regulator, was downregulated after induction of miR-663a mimic and upregulated with miR663a inhibitor treatment. These results suggested that miR-663a suppresses cell growth and invasion through inhibition of Cyclins and MMP9 expression.

AP-1 signaling was reported to regulate cyclin D1, cyclin E and MMP9 in various cancers including NSCLC $[7,21]$. Using luciferase reporter plasmid, we found that miR-663a inhibited AP-1 transcription activity. So among the potential miR-663a targets list predicted by TargetScan Human database, we sparked a strong interested in JunD which is a functional component of the AP1 transcription factor complex and also a proved target of miR-663a in human THP-1 monocytic cell [15]. In vitro overexpression of miR-663a significantly decreased JunD mRNA and protein expression compared to controls, whereas inhibition of miR-663a led to increased JunD mRNA and protein. Correspondingly, we also found a negative correlation between miR-663a and JunD mRNA in NSCLC tissues. Furthermore, fluorescent reporter assays demonstrated that miR-663a directly bound to the JunD 3'-UTR region, which was in accordance with previous report [15]. As an AP-1 transcription factor, JunD was reported to accelerate growth, inhibit apoptosis and enhance cancer cell invasion [22-26]. Using siRNA knockdown, we confirmed that JunD downregulation decreased expression of cyclin D1, cyclin E and MMP9 in lung cancer cells. JunD siRNA also abrogated the effect of miR-663a inhibitor. Thus our results indicated that miR-663a inhibited cell cycle and invasion, at least partly, through regulation of JunD expression.

\section{Conclusions}

In conclusion, miR-663a, a downregulated miRNA in NSCLC, was associated with lymph node metastasis. In addition, miR-663a regulated cell cycle and invasion by targeting AP-1 component JunD, which provides new insights into the molecular mechanisms of lung cancer progression.

\section{Ethics approval and consent to participate}

This study was conducted with the approval of the Ethics Committee at Shengjing Hospital of China Medical University. Written informed consent was obtained from all patients. Research carried out is in compliance with the Helsinki Declaration.

\section{Consent for publication \\ Not applicable}

\section{Availability of data and materials}

The dataset supporting the conclusions of this article is available in the following repository: http://pan.baidu.$\mathrm{com} / \mathrm{s} / 1 \mathrm{i} 4 \mathrm{YrsrF}$

\section{Additional files}

Additional file 1: Figure S1. A. Colony number of $\mathrm{H} 460$ cells transfected with a inhibitor was higher than cells transfected with control (control 415 \pm 32 vs mimic $581 \pm 17, p<0.001$ ). Colony number of H1299 transfected with miR-663a mimic was lower than cells transfected with control (control $278 \pm 20$ vs inhibitor $169 \pm 18, p<0.001$ ). B. Matrigel invasion assay showed that invading number of $\mathrm{H} 460$ transfected with miR-663a inhibitor was higher that cells transfected with control (control $45 \pm 3$ vs mimic $61 \pm 4, p=0.007$ ). Invading number of $\mathrm{H} 1299$ transfected with miR-663a mimic was lower that cells transfected with control (control $72 \pm 3$ vs inhibitor $46 \pm 4, p<0.001)$. C. Western blot showed that cyclin D1, cyclin E, MMP9, JunD protein levels of $\mathrm{H} 460$ cells treated with miR-663a inhibitor were higher than those of control. cyclin D1, cyclin E, MMP9, JunD protein levels of $\mathrm{H} 1299$ cells treated with miR-663a mimic were lower than those of control. D. Realtime RT-PCR showed that cyclin D1, cyclin E, MMP9, JunD mRNA levels of H460 cells treated with miR-663a inhibitor were higher than those of control. cyclin D1, cyclin E, MMP9, JunD mRNA levels of H1299 cells treated with miR-663a mimic were lower than those of control. Experiments were repeated in triplicate. Error bars indicate standard deviation. (TIFF $1946 \mathrm{~kb}$ )

Additional file 2: Figure S2. The JunD information from the TargetScan Human database. The information is from the TargetScan Human database. (TIFF $42 \mathrm{~kb}$ )

\section{Abbreviations}

3'-UTR: three prime untranslated region; miRNA: microRNA; NSCLC: non-small cell lung; RT-qPCR: reverse transcription quantitative polymerase chain reaction.

\section{Competing interests}

The authors declare that they have no competing interests.

\section{Authors' contributions}

$Y Z$ and $X X$ wrote the manuscript. $M Z, X W, X B$ and LK performed the cell experiments. $\mathrm{HL}$ and $Y Z$ performed the realtime $P C R$ analysis. $Y Z, H N$ and $P H$ participated in the design of the study and performed the statistical analysis. $\mathrm{PH}$ conceived of the study. All authors read and approved the final manuscript.

\section{Acknowledgement}

The study was supported by the National Natural Science Foundation of China (No. 81201833).

\section{Author details}

${ }^{1}$ Department of Geriatrics, Shengjing Hospital of China Medical University, 36 Sanhao Road, Shenyang 110004, China. ${ }^{2}$ Department of Respiratory Medicine, Shengjing Hospital of China Medical University, Shenyang, China.

Received: 21 February 2015 Accepted: 11 May 2016

Published online: 16 May 2016

\section{References}

1. Siegel R, Naishadham D, Jemal A. Cancer statistics, 2013. CA Cancer J Clin. 2013;63(1):11-30.

2. Minna JD, Roth JA, Gazdar AF. Focus on lung cancer. Cancer Cell. 2002;1(1): 49-52.

3. Schiller JH, Harrington D, Belani CP, Langer C, Sandler A, Krook J, Zhu J Johnson DH, Eastern Cooperative Oncology G. Comparison of four chemotherapy regimens for advanced non-small-cell lung cancer. $\mathrm{N}$ Engl J Med. 2002;346(2):92-8.

4. Chambers AF, Groom AC, MacDonald IC. Dissemination and growth of cancer cells in metastatic sites. Nat Rev Cancer. 2002;2(8):563-72.

5. Fidler IJ. The pathogenesis of cancer metastasis: the 'seed and soil' hypothesis revisited. Nat Rev Cancer. 2003;3(6):453-8.

6. Dong QZ, Wang Y, Dong XJ, Li ZX, Tang ZP, Cui QZ, Wang EH. CIP2A is overexpressed in non-small cell lung cancer and correlates with poor prognosis. Ann Surg Oncol. 2011;18(3):857-65.

7. Dong QZ, Wang Y, Tang ZP, Fu L, Li QC, Wang ED, Wang EH. Derlin-1 is overexpressed in non-small cell lung cancer and promotes cancer cell 
invasion via EGFR-ERK-mediated up-regulation of MMP-2 and MMP-9. Am J Pathol. 2013;182(3):954-64.

8. Lu J, Getz G, Miska EA, Alvarez-Saavedra E, Lamb J, Peck D, Sweet-Cordero A, Ebert BL, Mak RH, Ferrando AA et al. MicroRNA expression profiles classify human cancers. Nature. 2005;435(7043):834-8.

9. Yanaihara N, Caplen N, Bowman E, Seike M, Kumamoto K, Yi M, Stephens RM, Okamoto A, Yokota J, Tanaka T et al. Unique microRNA molecular profiles in lung cancer diagnosis and prognosis. Cancer Cell. 2006;9(3):189-98.

10. Takamizawa J, Konishi H, Yanagisawa K, Tomida S, Osada H, Endoh H, Harano T, Yatabe $Y$, Nagino M, Nimura $Y$ et al. Reduced expression of the let-7 microRNAs in human lung cancers in association with shortened postoperative survival. Cancer Res. 2004;64(11):3753-6.

11. Yu SL, Chen HY, Chang GC, Chen CY, Chen HW, Singh S, Cheng CL, Yu CJ, Lee $Y C$, Chen HS et al. MicroRNA signature predicts survival and relapse in lung cancer. Cancer Cell. 2008;13(1):48-57.

12. Ni CW, Qiu H, Jo H. MicroRNA-663 upregulated by oscillatory shear stress plays a role in inflammatory response of endothelial cells. Am J Physiol Heart Circ Physiol. 2011;300(5):H1762-1769.

13. Jian P, Li ZW, Fang TY, Jian W, Zhuan Z, Mei LX, Yan WS, Jian N. Retinoic acid induces $\mathrm{HL}-60$ cell differentiation via the upregulation of miR-663. J Hematol Oncol. 2011:4:20.

14. Pan J, Hu H, Zhou Z, Sun L, Peng L, Yu L, Sun L, Liu J, Yang Z, Ran Y. Tumor-suppressive mir-663 gene induces mitotic catastrophe growth arrest in human gastric cancer cells. Oncol Rep. 2010;24(1):105-12.

15. Tili E, Michaille JJ, Adair B, Alder H, Limagne E, Taccioli C, Ferracin M, Delmas D, Latruffe N, Croce CM. Resveratrol decreases the levels of miR-155 by upregulating miR-663, a microRNA targeting JunB and JunD. Carcinogenesis. 2010;31(9):1561-6.

16. Tili E, Michaille JJ, Alder H, Volinia S, Delmas D, Latruffe N, Croce CM. Resveratrol modulates the levels of microRNAs targeting genes encoding tumor-suppressors and effectors of TGFbeta signaling pathway in SW480 cells. Biochem Pharmacol. 2010;80(12):2057-65.

17. Yan-Fang T, Jian N, Jun L, Na W, Pei-Fang X, Wen-Li Z, Dong W, Li P, Jian W, Xing $\mathrm{F}$ et al. The promoter of miR-663 is hypermethylated in Chinese pediatric acute myeloid leukemia (AML). BMC Med Genet. 2013;14:74.

18. Hu H, Li S, Cui X, Lv X, Jiao Y, Yu F, Yao H, Song E, Chen Y, Wang M et al. The overexpression of hypomethylated miR-663 induces chemotherapy resistance in human breast cancer cells by targeting heparin sulfate proteoglycan 2 (HSPG2). J Biol Chem. 2013;288(16):10973-85.

19. Yi C, Wang Q, Wang L, Huang Y, Li L, Liu L, Zhou X, Xie G, Kang T, Wang $\mathrm{H}$ et al. MiR-663, a microRNA targeting p21(WAF1/CIP1), promotes the proliferation and tumorigenesis of nasopharyngeal carcinoma. Oncogene. 2012;31(41):4421-33.

20. Shi Y, Chen C, Zhang X, Liu Q, Xu JL, Zhang HR, Yao XH, Jiang T, He ZC, Ren $Y$ et al. Primate-specific miR-663 functions as a tumor suppressor by targeting PIK3CD and predicts the prognosis of human glioblastoma. Clin Cancer Res. 2014;20(7):1803-13.

21. Kharman-Biz A, Gao H, Ghiasvand R, Zhao C, Zendehdel K, Dahlman-Wright K. Expression of activator protein-1 (AP-1) family members in breast cancer. BMC Cancer. 2013;13:441.

22. Castellazzi M, Spyrou G, La Vista N, Dangy JP, Piu F, Yaniv M, Brun G. Overexpression of $c-j u n$, junB, or junD affects cell growth differently. Proc Natl Acad Sci U S A. 1991;88(20):8890-4.

23. Kobierski LA, Chu HM, Tan Y, Comb MJ. cAMP-dependent regulation of proenkephalin by JunD and JunB: positive and negative effects of AP-1 proteins. Proc Natl Acad Sci U S A. 1991;88(22):10222-6.

24. Agarwal SK, Novotny EA, Crabtree JS, Weitzman JB, Yaniv M, Burns AL, Chandrasekharappa SC, Collins FS, Spiegel AM, Marx SJ. Transcription factor JunD, deprived of menin, switches from growth suppressor to growth promoter. Proc Natl Acad Sci U S A. 2003:100(19):10770-5.

25. Kook SH, Son YO, Jang YS, Lee KY, Lee SA, Kim BS, Lee HJ, Lee JC. Inhibition of c-Jun N-terminal kinase sensitizes tumor cells to flavonoid-induced apoptosis through down-regulation of JunD. Toxicol Appl Pharmacol. 2008;227(3):468-76.

26. Chen HW, Lee JY, Huang JY, Wang CC, Chen WJ, Su SF, Huang CW, Ho CC, Chen JJ, Tsai MF et al. Curcumin inhibits lung cancer cell invasion and metastasis through the tumor suppressor HLJ1. Cancer Res. 2008;68(18): 7428-38.

\section{Submit your next manuscript to BioMed Central and we will help you at every step:}

- We accept pre-submission inquiries

- Our selector tool helps you to find the most relevant journal

- We provide round the clock customer support

- Convenient online submission

- Thorough peer review

- Inclusion in PubMed and all major indexing services

- Maximum visibility for your research

Submit your manuscript at www.biomedcentral.com/submit

) Biomed Central 